\title{
Optimal strategy for controlling transport in inertial Brownian motors
}

\author{
Lukasz Machura ${ }^{1,2}$, Marcin Kostur ${ }^{1}$, Fabio Marchesoni ${ }^{3}$, \\ Peter Talkner ${ }^{1}$, Peter Hänggi ${ }^{1}$ and Jerzy Luczka ${ }^{2}$ \\ ${ }^{1}$ Institute of Physics, University of Augsburg, Universitätsstrasse 1, D-86135 Augsburg, \\ Germany \\ ${ }^{2}$ Institute of Physics, University of Silesia, P-40-007 Katowice, Poland \\ ${ }^{3}$ Dipartimento di Fisica, Università di Camerino, I-62032 Camerino, Italy \\ E-mail: lukasz.machura@physik.uni-augsburg.de
}

Received 9 May 2005, in final form 3 August 2005

Published 4 November 2005

Online at stacks.iop.org/JPhysCM/17/S3741

\begin{abstract}
In order to optimize the directed motion of an inertial Brownian motor, we identify the operating conditions that both maximize the motor current and minimize its dispersion. Extensive numerical simulation of an inertial rocked ratchet displays that two quantifiers, namely the energetic efficiency and the Péclet number (or equivalently the Fano factor), suffice to determine the regimes of optimal transport. The effective diffusion of this rocked inertial Brownian motor can be expressed as a generalized fluctuation theorem of the Green-Kubo type.
\end{abstract}

(Some figures in this article are in colour only in the electronic version)

\section{Introduction}

The theoretical concepts of Brownian motors and ratchet transport [1] have been experimentally realized in a variety of systems. Examples are cold atoms in optical lattices [2], colloidal particles in holographic optical trapping patterns [3], ratchet cellular automata [4], superconducting films with periodic arrays of asymmetric pinning sites [5, 6], to mention only a few.

When we study the motion of Brownian motors, the natural transport measure is a conveniently defined average asymptotic velocity $\langle v\rangle$ of the Brownian motors. It describes how much time the typical particle needs to overcome a given distance in the asymptotic (long-time) regime. This velocity, however, is not the only relevant transport criterion. Other attributes can also be important. In order to establish these, we consider the two following aspects: the quality of the transport and the energetic efficiency of such a system.

In figure 1, one can identify two different groups $\mathrm{A}$ and $\mathrm{B}$ of random trajectories of the Brownian particle; both possess the same average drift velocity $\langle v\rangle$. However, it is obvious 


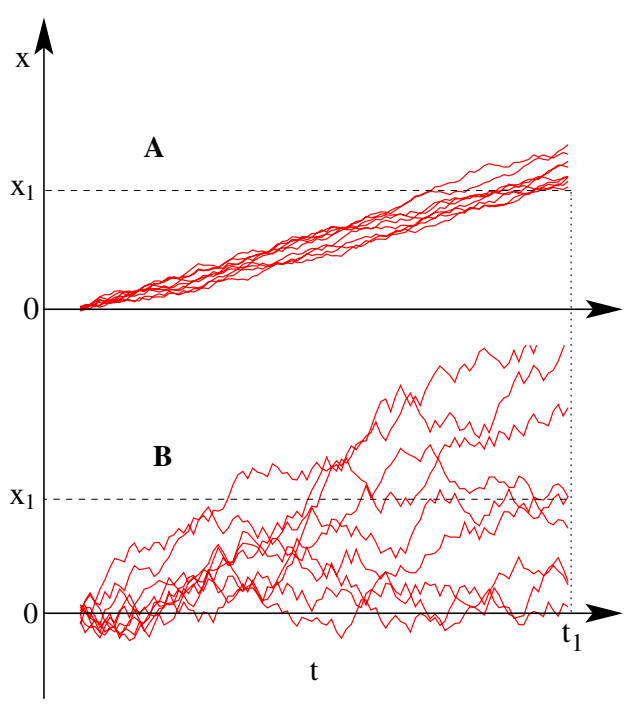

Figure 1. Two sets of illustrative trajectories of an inertial, rocked Brownian motor (see in text). Both sets of trajectories A and B possess the same average asymptotic velocity, but exhibit a distinct different diffusion behaviour.

upon inspection that the dynamical properties of these two groups of trajectories are different. The particles from group A travel more or less coherently together while the particles from group B spread out as time goes by. If we fix the distance $x=x_{1}$ then most particles from group A reach this distance at about the same time $t=t_{1}$, while for $t=t_{1}$ most of the B trajectories either stay behind or have already proceeded to more distant positions. It is thus evident that the noise-assisted, directed transport for the particles in group A is more effective than in group B.

There is still another efficiency aspect related to Brownian motor transport. This refers to the external energy input into the system which may be essential in practical applications. We like to know how much of this input energy is converted into useful work, namely into directed cargo transport, and how much of it gets wasted. Since motors move in a dissipative environment, we need to know how much of the input energy is being spent for moving a certain distance against the acting friction force. Figure 2 depicts trajectories representing different motor scenarios. Motor $\mathrm{C}$ moves forward unidirectionally, while motor D moves in a more complicated manner: its motion alternates small oscillations and fast episodes, mostly in the forward direction, but sometimes also in the backward direction. Again the mean velocity in both cases is the same; however, particle $\mathrm{C}$ uses energy pumped from the environment to proceed constantly forward while particle D wastes part of its energy to perform oscillations and back-turns. By simply inspecting these schematic pictures one can guess immediately when directed transport is more effective.

We note that, in figure 1, the cases A and B can be characterized by the effective diffusion coefficient $D_{\text {eff }}$, i.e., by the spreading of fluctuations in the position space, while the cases C and $\mathrm{D}$ in figure 2 can be characterized by the variance of velocity $\sigma_{\mathrm{v}}^{2}=\left\langle v^{2}\right\rangle-\langle v\rangle^{2}$. The three quantities $\langle v\rangle, D_{\text {eff }}$ and $\sigma_{\mathrm{v}}^{2}$ can be combined to define two important characteristics of transport, namely the efficiency of noise rectification and the so-called Péclet number [7].

Our work is organized as follows. In the following section, we detail the model of an inertial rocked Brownian motor. In section 3, we present a general discussion of the efficiency 


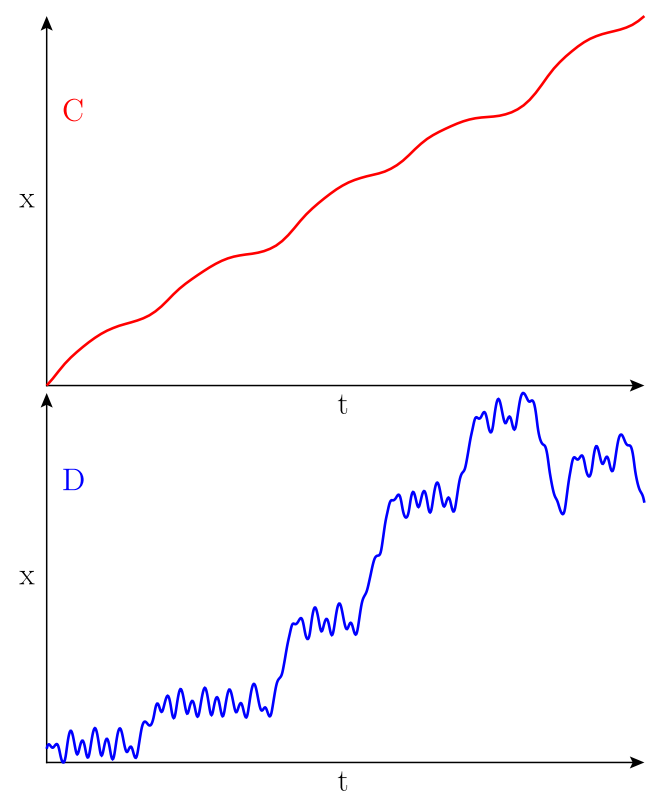

Figure 2. Typical trajectories of an inertial, rocking Brownian motor; both sets assume the same average velocity but differing velocity fluctuations.

measures of Brownian motors. In section 4, a description of the ratchet based on point processes is introduced. In sections 5 and 6, our numerical findings are analysed in the context of the optimization conditions for transport of inertial Brownian motors. A summary is provided in section 7 .

\section{Inertial rocked Brownian motors}

The archetype of the inertial Brownian motor is represented by a classical particle of mass $m$ moving in a spatially periodic and asymmetric potential $V(x)=V(x+L)$ with period $L$ and barrier height $\Delta V[8,9]$. The particle is driven by an external, unbiased, time-periodic force of amplitude $A$ and angular frequency $\Omega$ (or period $\mathcal{T}_{0}=2 \pi / \Omega$ ). The system is additionally subjected to thermal noise $\xi(t)$. The dynamics of the system is modelled by the Langevin equation [10]

$$
m \ddot{x}+\gamma \dot{x}=-V^{\prime}(x)+A \cos (\Omega t)+\sqrt{2 \gamma k_{\mathrm{B}} T} \xi(t),
$$

where a dot denotes differentiation with respect to time and a prime denotes a differentiation with respect to the Brownian motor coordinate $x$. The parameter $\gamma$ denotes the Stokes friction coefficient, $k_{\mathrm{B}}$ is the Boltzmann constant and $T$ is the temperature. The thermal fluctuations due to the coupling of the particle with the environment are modelled by a zero-mean, Gaussian white noise $\xi(t)$ with auto-correlation function $\langle\xi(t) \xi(s)\rangle=\delta(t-s)$ satisfying Einstein's fluctuation-dissipation relation.

Upon introducing characteristic length scale and time scale, equation (1) can be rewritten in dimensionless form, namely

$$
\ddot{\hat{x}}+\hat{\gamma} \dot{\hat{x}}=-\hat{V}^{\prime}(\hat{x})+a \cos (\omega \hat{t})+\sqrt{2 \hat{\gamma} D_{0}} \hat{\xi}(\hat{t})
$$

with [11]

$$
\hat{x}=\frac{x}{L}, \quad \hat{t}=\frac{t}{\tau_{0}}, \quad \tau_{0}^{2}=\frac{m L^{2}}{\Delta V} .
$$




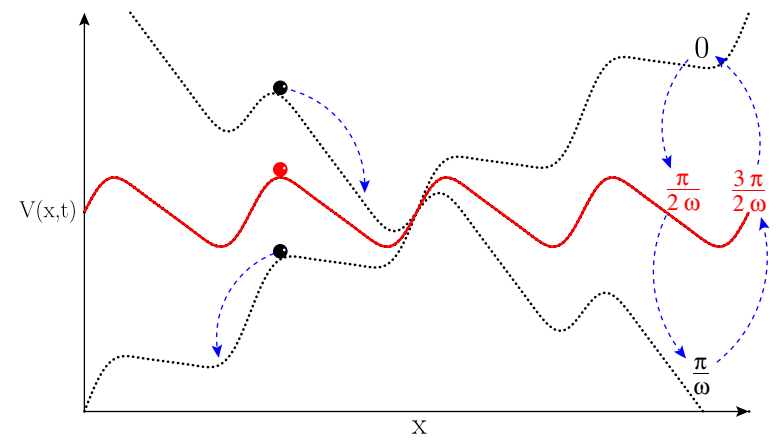

Figure 3. Schematic picture of a rocking ratchet with the potential $V(x, t)=V(x)-x a \cos (\omega t)$; cf equations (2) and (4).

The characteristic time $\tau_{0}$ is the time a particle of mass $m$ needs to move a distance $L / 2$ under the influence of the constant force $\Delta V / L$ when starting with velocity zero. The remaining rescaled parameters are:

- the friction coefficient $\hat{\gamma}=(\gamma / m) \tau_{0}=\tau_{0} / \tau_{L}$ is the ratio of the two characteristic times, $\tau_{0}$ and the relaxation time of the velocity degree of freedom, i.e., $\tau_{L}=m / \gamma$,

- the potential $\hat{V}(\hat{x})=V(x) / \Delta V=\hat{V}(\hat{x}+1)$ has unit period and unit barrier height $\Delta \hat{V}=1$,

- the amplitude $a=A L / \Delta V$ and the frequency $\omega=\Omega \tau_{0}$ (or the period $\left.\mathcal{T}=2 \pi / \omega\right)$,

- the zero-mean white noise $\hat{\xi}(\hat{t})$ has auto-correlation function $\langle\hat{\xi}(\hat{t}) \hat{\xi}(\hat{s})\rangle=\delta(\hat{t}-\hat{s})$ with rescaled noise intensity $D_{0}=k_{\mathrm{B}} T / \Delta V$.

From now on, we will use only the dimensionless variables and omit the 'hat' for all quantities in equation (2).

For the asymmetric ratchet potential $V(x)$ we consider a linear superposition of three spatial harmonics [11],

$$
V(x)=V_{0}\left[\sin (2 \pi x)+c_{1} \sin (4 \pi x)+c_{2} \sin (6 \pi x)\right],
$$

where $V_{0}$ normalizes the barrier height to unity and the parameters $c_{1}$ and $c_{2}$ determine the ratchet profile. Below, we analyse the case when $c_{1}=0.245$ and $c_{2}=0.04$. Then $V_{0}=0.461$. This potential is shown as a bold (red) line in figure 3 .

\section{Quantifiers characterizing optimal transport of Brownian motors}

As already elucidated above, there are several quantities that characterize the effectiveness of directed transport. The effective diffusion coefficient, describing the fluctuations around the average position of the particles, is defined as

$$
D_{\text {eff }}=\lim _{t \rightarrow \infty} \frac{\left\langle x^{2}(t)\right\rangle-\langle x(t)\rangle^{2}}{2 t},
$$

where the brackets $\langle\cdots\rangle$ denote an average over the initial conditions of position and velocity and over all realizations of the thermal noise. The coefficient $D_{\text {eff }}$ can also be introduced via a generalized Green-Kubo relation which we detail in the appendix. Intuitively, if the stationary velocity is large and the spread of trajectories is small, the diffusion coefficient is small and 
the transport is more effective. To quantify this, we can introduce the dimensionless Péclet number $P e[7,12]$ by use of a double-averaging procedure, i.e.,

$$
P e=\frac{L\langle\langle v\rangle\rangle}{D_{\mathrm{eff}}},
$$

where the 'double-average' $\langle\langle v\rangle\rangle$ denotes the average of the asymptotic velocity over one cycle of the external drive, i.e.,

$$
\langle\langle v\rangle\rangle=\lim _{t \rightarrow \infty} \frac{1}{t} \int_{0}^{t}\left\langle v\left(t^{\prime}\right)\right\rangle \mathrm{d} t^{\prime}=\frac{\omega}{2 \pi} \int_{0}^{2 \pi / \omega}\left\langle v\left(t^{\prime}\right)\right\rangle_{\text {as }} \mathrm{d} t^{\prime},
$$

where the average $\langle\cdots\rangle_{\text {as }}$ in the second integral refers to the asymptotic periodic state.

Originally, the Péclet number $P e$ arises in problems of heat transfer in fluids where it stands for the ratio of heat advection to diffusion. When the Péclet number is small, the random motion dominates; when it is large, the ordered and regular motion dominates. The value of the Péclet number depends on some characteristic length scale of the system. Dealing with ratchets, the most adequate choice for such a length scale is the period of the periodic potential, which in rescaled units is equal to 1.

The second aspect of the motor trajectories we want to control has to do with the fluctuations of the velocity $v(t)$. In the long-time regime, it is characterized by the variance $\sigma_{\mathrm{v}}^{2}=\left\langle\left\langle v^{2}\right\rangle\right\rangle-\langle\langle v\rangle\rangle^{2}$. The Brownian motor moves with an actual velocity $v(t)$, which is typically contained within the interval

$$
v(t) \in\left(\langle\langle v\rangle\rangle-\sigma_{\mathrm{v}},\langle\langle v\rangle\rangle+\sigma_{\mathrm{v}}\right) .
$$

Now, if $\sigma_{\mathrm{v}}>\langle\langle v\rangle\rangle$, the Brownian motor may possibly move for some time in the direction opposite to its average velocity $\langle\langle v\rangle\rangle$ and the directed transport becomes less efficient. If we want to optimize the effectiveness of the motor motion we must introduce a measure for the efficiency $\eta$ that accounts for the velocity fluctuations, too, namely [13]

$$
\eta=\frac{\langle\langle v\rangle\rangle^{2}}{\mid\left\langle\langle v\rangle^{2}+\sigma_{\mathrm{v}}^{2}-D_{0}\right|}=\frac{\langle\langle v\rangle\rangle^{2}}{\left|\left\langle v^{2}\right\rangle\right\rangle-D_{0} \mid} .
$$

This definition follows from an energy balance of the underlying equation of motion (2) (see the appendix of [11]). If the variance of velocity $\sigma_{\mathrm{v}}$ is reduced, the energetic efficiency (9) increases and the transport of the Brownian motor becomes more efficient.

\section{A corresponding point process related to the rocked Brownian motor dynamics}

The running trajectories can be characterized in a coarse-grained way by only counting the events when a trajectory traverses from one potential well into a neighbouring one, and by disregarding the details of the intra-well motion, see figure 4. In this way, a point process can be introduced that can be investigated in a standard way [14]. Most, though not all, of the quantities describing the original continuous process can be retrieved from the so-defined point process. For this purpose we introduce two random, natural numbers $N_{k}^{\alpha}$, where $\alpha=\{\mathrm{R}, \mathrm{L}\}$ stands for right $(\mathrm{R})$ and left $(\mathrm{L})$. The number $N_{k}^{\mathrm{R}}$ is given by the number of barrier crossings towards the right within the $k$ th period of the driving, i.e., in the time between $(k-1) \mathcal{T}$ and $k \mathcal{T}$. The respective number of barrier crossings to the left is denoted by $N_{k}^{\mathrm{L}}$. The difference

$$
N_{k}=N_{k}^{\mathrm{R}}-N_{k}^{\mathrm{L}}
$$

indicates that during a temporal period $\mathcal{T}$ the particle has covered the distance $x_{k}=N_{k} L=N_{k}$. Hence the average, asymptotic velocity is given by 


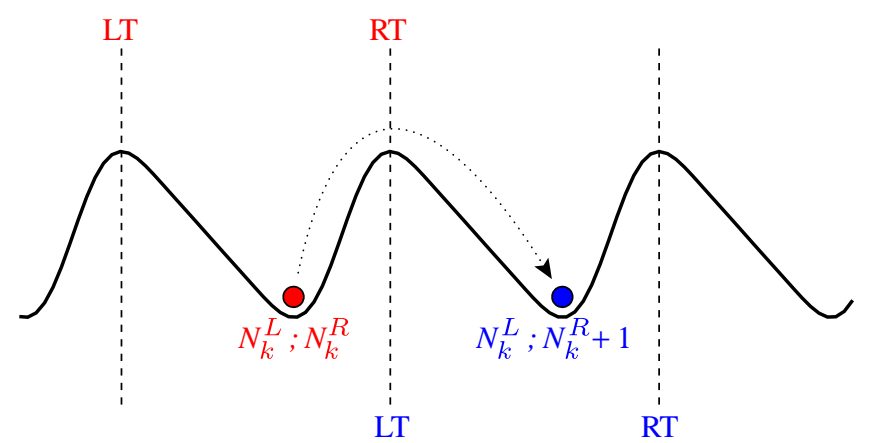

Figure 4. The point process related to rocked Brownian motor dynamics. Two thresholds LT and RT are the corresponding maxima, to the left and to the right of the particle position. If the particle jumps over the right neighbouring barrier, the number $N_{k}^{\mathrm{R}}$ increases to $N_{k}^{\mathrm{R}}+1$ and the previous right-sided threshold assumes the role of the left-sided threshold.

$$
\begin{aligned}
\langle\langle v\rangle\rangle=\lim _{t \rightarrow \infty} & \frac{1}{t} \int_{0}^{t}\left\langle v\left(t^{\prime}\right)\right\rangle \mathrm{d} t^{\prime}=\lim _{K \rightarrow \infty} \frac{1}{K \mathcal{T}} \sum_{k=1}^{K} \int_{(k-1) \mathcal{T}}^{k \mathcal{T}}\left\langle v\left(t^{\prime}\right)\right\rangle \mathrm{d} t^{\prime} \\
& =\lim _{K \rightarrow \infty} \frac{1}{K \mathcal{T}} \sum_{k=1}^{K} x_{k}=\lim _{K \rightarrow \infty} \frac{1}{\mathcal{T} K} \sum_{k=1}^{K} N_{k}=\frac{\langle N\rangle}{\mathcal{T}} .
\end{aligned}
$$

Analogously, the effective diffusion coefficient is determined by the relation

$$
D_{\text {eff }}=\frac{\left\langle\delta N^{2}\right\rangle}{2 \mathcal{T}}=\frac{\left\langle N^{2}\right\rangle-\langle N\rangle^{2}}{2 \mathcal{T}} .
$$

A related quantity is the Fano factor $F$ [15], defined here as the fluctuation to the first moment ratio

$$
F=\frac{\left\langle\delta N^{2}\right\rangle}{\langle N\rangle}
$$

As such, the Fano factor provides a quantitative measure of the relative number fluctuations or the relative randomness of the process; in the case of a Poisson process $F=1$.

On the other hand, from (6), (11) and (12) it follows that the Péclet number can be expressed as

$$
P e=\frac{2\langle N\rangle}{\left\langle\delta N^{2}\right\rangle} .
$$

This quantifier is thus related to the Fano factor via the relation $P e=2 / F$.

\section{Numerical analysis}

The noiseless, deterministic inertial rocked ratchet shows a rather complex behaviour and, in distinct contrast to overdamped rocked Brownian motors [16], often exhibits a chaotic dynamics $[8,17]$. By adding noise, one typically activates a diffusive dynamics, thus allowing for stochastic escape events among possibly coexisting attractors. As analytical methods to handle these situations effectively do not exist, we carried out extensive numerical simulations. We have numerically integrated equation (2) by the Euler method with time step $h=5 \times 10^{-4} \mathcal{T}$. The initial conditions for the coordinate $x(t)$ were chosen according to a uniform distribution within one cell of the ratchet potential. The starting velocities of the particles were also distributed uniformly in the interval $[-0.2,0.2]$. 
(a)

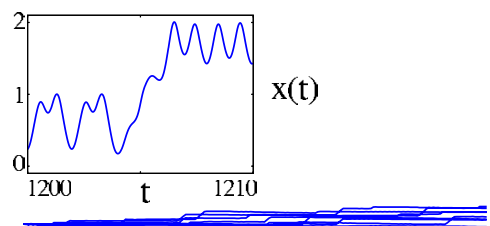

$a=3.29$

(b)

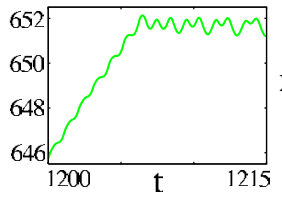

$\mathrm{x}(\mathrm{t})$

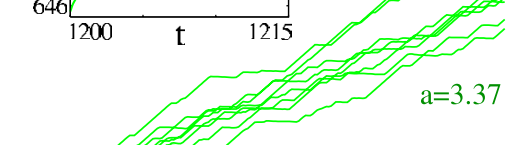

(c)

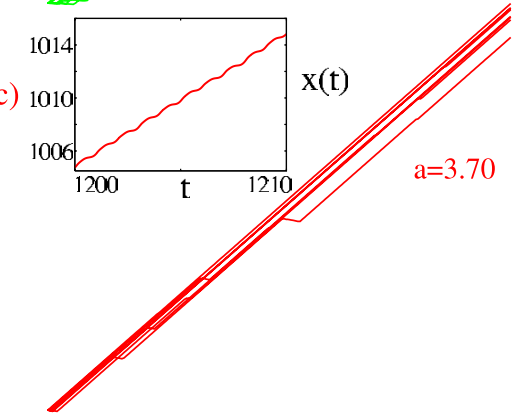

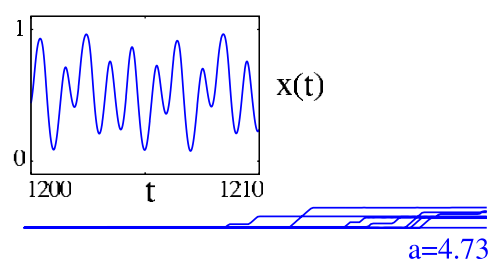

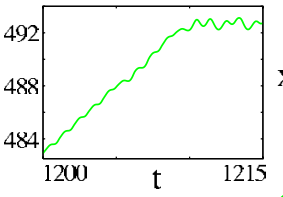

$\mathrm{x}(\mathrm{t})$
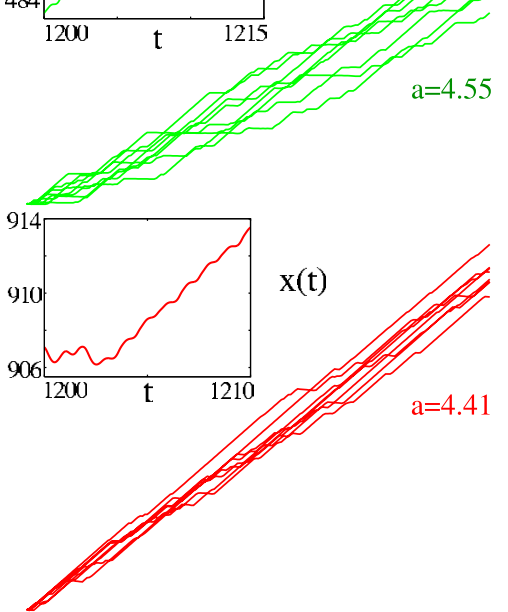

Figure 5. Brownian trajectories of the rocked particle moving in the asymmetric ratchet potential $V(x)=V_{0}[\sin (2 \pi x)+0.245 \sin (4 \pi x)+0.04 \sin (6 \pi x)]$, where $V_{0} \simeq 0.461$ normalizes the barrier height to unity. The forces stemming from such a potential range between -4.67 and 1.83 . The two angular frequencies at the well bottom and at the barrier top are the same, reading 5.34. The remaining parameters are: $\gamma=0.9, \omega=4.9$ and $D_{0}=0.001$. The values of the driving amplitude are $a=3.29,3.37,3.70,4.41,4.55,4.73$. One can see that for $a=3.29$ and 4.73 in part (a) (in blue online), the particles usually oscillate in a potential well, most of the time performing only a few steps. This results in an almost zero mean velocity, a very small effective diffusion but with rather large velocity fluctuations. For another set of driving amplitudes: $a=3.37$ and 4.55 in part (b) (in green online) the mean velocity is large, $\sigma_{\mathrm{v}}$ becomes suppressed, but the effective diffusion exhibits an enlargement due to a 'battle between attractors'. Part (c): the cases $a=3.70$ and $a=4.41$ (in red online) correspond to the optimal modus operandi of the inertial Brownian motor- - the net drift is maximal and fluctuations get suppressed.

The first $10^{3}$ periods $\mathcal{T}$ of the external force were skipped in order to avoid transient effects. We employed two tactics of extracting the above characteristics from the generated trajectories. For the estimation of the energetic efficiency (or velocity fluctuations) the usual averages over time $\left(10^{5} \mathcal{T}\right)$ and 333 different realizations were taken. In the case of the Péclet number (or effective diffusion) we used the point process approach (see previous section for details); therefore, only one time average of long-time runs $\left(10^{6} \mathcal{T}\right)$ was required.

Typically, there are two possible dynamical states of the ratchet system: a locked state, in which the particle oscillates mostly within one potential well (cf the case with amplitude $a=4.73$ in figure 5), and a running state, in which the particles surmount the barriers of the potential. Moreover, one can distinguish two classes of running states: either the particle overcomes the barriers without any back-turns (cf the case with an amplitude $a=3.70$ in 


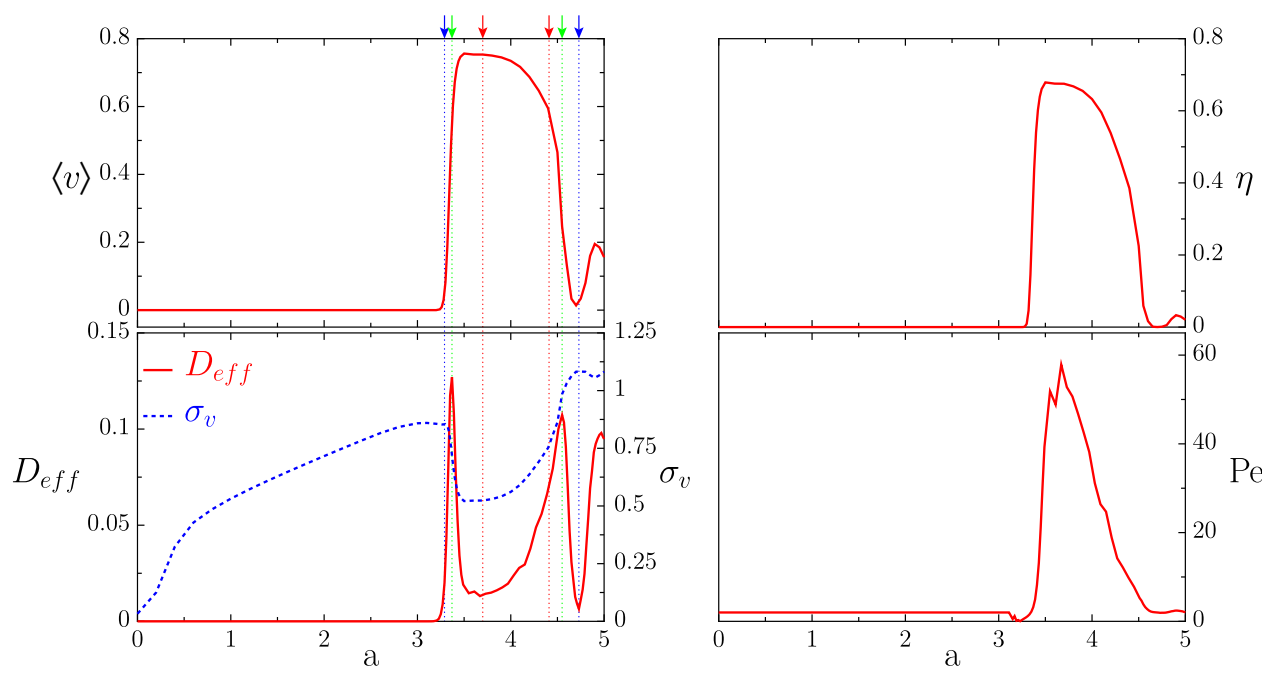

Figure 6. Left top: the average, dimensionless velocity $\langle v\rangle$ of the inertial, rocked Brownian motor under non-adiabatic driving conditions. Left bottom: corresponding velocity fluctuations $\sigma_{\mathrm{v}}$ (dotted line) and corresponding diffusion coefficient $D_{\text {eff }}$ (solid line). Right top: Brownian motor efficiency $\eta$. Right bottom: the Péclet number $P e$, being proportional the inverse of the Fano factor. All quantities are plotted versus the external driving amplitude $a$. Values of the remaining parameters are the same as in figure 5; e.g. the thermal noise strength here is $D_{0}=0.001$. The numerical errors are within the line width.

figure 5) or it undergoes frequent oscillations and back-scattering events (cf the case with amplitude $a=4.55$ in figure 5). For a small driving amplitude, we find that the locked behaviour is generic, implying that the average motor velocity is almost zero; see figure 5 . If the amplitude is increased up to some critical value, here $a=3.25$, the running solutions emerge. Around that critical point, there occurs a 'battle of attractors' and the particle burns energy for both barrier crossings and intra-well oscillations. This behaviour is reflected in an enormous enhancement of the effective diffusion [18].

If the driving amplitude is further increased, a regime of optimal transport sets in. The rapid growth of the average velocity is accompanied by a decline of both the position and the velocity fluctuations. This means that the trajectories bundle closely together; note the case $a=3.70$ in figure 5. Because there are no intra-well oscillations, the energy that gets dissipated per unit distance is minimal.

At even larger drive amplitudes an upper threshold is approached (in the present case this threshold is located at around $a=4.7$ ) where the velocity sharply decreases to a value close to zero. Moreover, the diffusion coefficient is small and the velocity fluctuations are large; cf the case with amplitude $a=4.73$ in figure 5. In this regime, the particle dangles around its actual position, as it occurs for $a<3$, meaning that its motion is confined mostly to one well. We note, however, that the amplitude of the intra-well oscillations becomes much larger, so the corresponding velocity fluctuations are also large.

We conclude that the diffusion coefficient is small for cases when the particle performs either locked motion or running motion without back-turns.

All these considerations are accurately encoded and described by the two previously discussed measures, namely, the efficiency (9) and the Péclet number $P e$ in (6) or in (14). It is found that the optimal regime for the ideal modus operandi of the Brownian motor is achieved when both the efficiency and the Péclet number become maximal; see in figure 6. Indeed, 

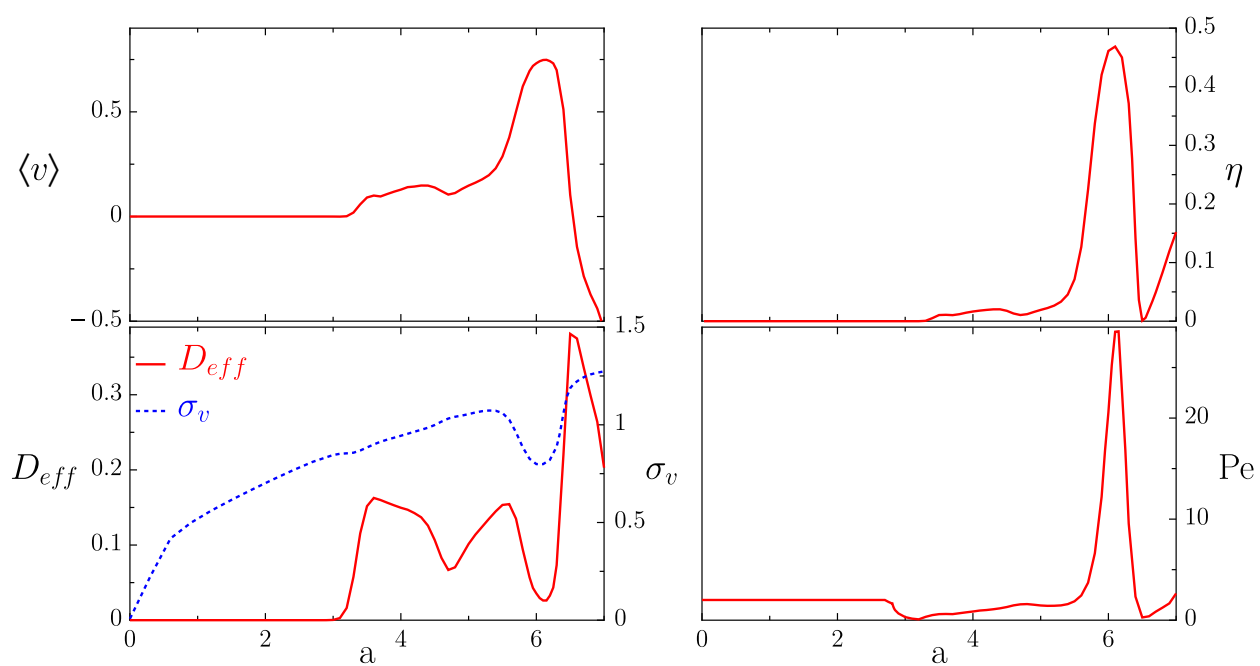

Figure 7. The same as in figure 6 but here for a five times larger temperature, i.e., $D_{0}=0.005$.

in this regime of optimal performance, the particle moves forward steadily, undergoing rare back-turns [19]; see the case $a=3.70$ in figure 5 .

\section{Role of temperature}

We next address the dependence on the strength of thermal noise. In figure 7, we present our numerical results for the noise-assisted, directed transport at a larger temperature, namely for $D_{0}=0.005$. The potential barrier height is still rather high in comparison to the thermal energy. In this regime, a so-called current reversal, i.e., a change of the transport direction, occurs as a function of the driving amplitude. Otherwise, the behaviour remains qualitatively the same as for lower noise $D=0.001$. The diffusion coefficient exhibits three maxima and two minima in the corresponding interval of the drive amplitudes. However, the optimal regime corresponds to the neighbourhood of the second minimum of the diffusion coefficient. In contrast, we notice that at lower noise $D=0.001$, the optimal regime set in within the neighbourhood of the first minimum of $D_{\text {eff }}$.

Under higher temperature operating conditions, optimal transport also occurs when both the efficiency $\eta$ and the Péclet number $P e$ are maximal.

\section{Summary}

In this work, criteria for the optimal transport of an inertial rocked ratchet were established using two characteristic quantifiers: the energetic efficiency (9) and the Péclet number (6). Adapting the methods of point processes to rocked Brownian motors, we expressed the averaged motor velocity and the position-diffusion coefficient by corresponding averages of the point process $N_{k}$. Both these measures can be obtained from simulations of the driven Langevin dynamics (2).

The Fano factor $F$ used in the theory of point processes is related to the Péclet number in a simple manner via $P e=2 / F$. In our case, it is more convenient to employ the Péclet number because in regimes where the average velocity is very small the Péclet number assumes values close to zero, while the Fano factor would diverge. From our numerical analysis it follows that 
the optimal modus operandi for the inertial Brownian motor is obtained when the efficiency $\eta$ and the Péclet number simultaneously assume maximal values.

\section{Acknowledgments}

The authors gratefully acknowledge financial support by the Deutsche Forschungsgemeinschaft via grant HA 1517/13-4, the Graduiertenkolleg 283 (LM, PT, PH), the collaborative research grant SFB 486, the DAAD-KBN (German-Polish project Stochastic Complexity) (PH and JÆ), and the ESF, Program Stochastic Dynamics: fundamentals and applications; STOCHDYN (PH and FM).

\section{Appendix}

In the present paper we have considered the effective diffusion coefficient, which is defined as

$$
D_{\text {eff }}=\lim _{t \rightarrow \infty} \frac{\left\langle x^{2}(t)\right\rangle-\langle x(t)\rangle^{2}}{2 t}
$$

where the brackets $\langle\cdots\rangle$ denote an average over the initial conditions of position and velocity and over all realizations of the thermal noise. Another definition of the diffusion coefficient is given by the formula

$$
D=\lim _{t \rightarrow \infty} \frac{\left\langle[\delta x(t)-\delta x(0)]^{2}\right\rangle}{2 t},
$$

where $\delta x(t)=x(t)-\langle x(t)\rangle$. By inspection one finds

$$
D_{\text {eff }}=D
$$

if

$$
\lim _{t \rightarrow \infty} \frac{1}{t}\langle\delta x(t) \delta x(0)\rangle=0 .
$$

In our case, this term vanishes because of the presence of thermal noise and dissipation. More generally, $|\langle\delta x(t) \delta x(0)\rangle|$ may increase at most as $t^{1 / 2}$ if the diffusion coefficient $D$ as defined in (16) is finite. Consequently, for such processes equation (17) also holds.

We now show that the diffusion constant $D$ is related to the auto-correlation function of the velocity via a Green-Kubo relation, in spite of the fact that the system is far from equilibrium. For a system with periodic driving, $D$ takes the form

$$
D=\int_{0}^{\infty} \mathrm{d} s \bar{C}(s)
$$

where

$$
\bar{C}(s)=\frac{1}{\mathcal{T}} \int_{0}^{\mathcal{T}} \mathrm{d} \tau C_{\mathrm{as}}(\tau, s)
$$

denotes the time average of the velocity correlation function $C_{\text {as }}(\tau, s)$ over one period $\mathcal{T}=2 \pi / \omega$ of the driving and where

$$
C_{\text {as }}(t, s)=\langle\delta v(t) \delta v(t+s)\rangle_{\text {as }}=\langle\delta v(t+s) \delta v(t)\rangle_{\text {as }}
$$

is the non-equilibrium asymptotic velocity-velocity correlation function. In the case of periodic driving, this function is periodic with respect to the first argument, i.e.,

$$
C(t, s)=C(t+\mathcal{T}, s) .
$$


To show the Green-Kubo relation, we start from the expression $\dot{x}(t)=v(t)$, from which it follows that

$$
\delta x(t)-\delta x(0)=\int_{0}^{t} \mathrm{~d} s \delta v(s) .
$$

Therefore (16) takes the form

$2 D=\lim _{t \rightarrow \infty} \frac{1}{t} \int_{0}^{t} \mathrm{~d} s_{1} \int_{0}^{t} \mathrm{~d} s_{2}\left\langle\delta v\left(s_{1}\right) \delta v\left(s_{2}\right)\right\rangle=\lim _{t \rightarrow \infty} \frac{1}{t} \int_{0}^{t} \mathrm{~d} s_{1} \int_{0}^{t} \mathrm{~d} s_{2} C\left(s_{2}, s_{1}-s_{2}\right)$,

where

$$
C(t, s)=\langle\delta v(t) \delta v(t+s)\rangle .
$$

Changing the integration variables $\left(s_{1}, s_{2}\right) \rightarrow\left(s=s_{1}-s_{2}, \tau=s_{2}\right)$ and exploiting the symmetry of the correlation function, $C(t, s)=C(t+s,-s)$, one obtains

$$
\begin{aligned}
D=\lim _{t \rightarrow \infty} \frac{1}{t} & \int_{0}^{t} \mathrm{~d} s \int_{0}^{t-s} \mathrm{~d} \tau C(\tau, s) \\
& =\lim _{t \rightarrow \infty} \frac{1}{t} \int_{0}^{t} \mathrm{~d} s \int_{0}^{t} \mathrm{~d} \tau C(\tau, s)-\lim _{t \rightarrow \infty} \frac{1}{t} \int_{0}^{t} \mathrm{~d} s \int_{t-s}^{t} \mathrm{~d} \tau C(\tau, s) .
\end{aligned}
$$

We assume that the diffusion coefficient is finite. Therefore the second term in the second line of (26) tends to zero as $t \rightarrow \infty$, so

$$
D=\int_{0}^{\infty} \mathrm{d} s \lim _{t \rightarrow \infty} \frac{1}{t} \int_{0}^{t} \mathrm{~d} \tau C(\tau, s)
$$

For $t=K \mathcal{T}$, one splits the second integral into sum over subsequent periods,

$\lim _{t \rightarrow \infty} \frac{1}{t} \int_{0}^{t} \mathrm{~d} \tau C(\tau, s)=\lim _{K \rightarrow \infty} \frac{1}{K \mathcal{T}} \sum_{k=1}^{K} \int_{(k-1) \mathcal{T}}^{k \mathcal{T}} \mathrm{d} \tau C(\tau, s)=\frac{1}{\mathcal{T}} \int_{0}^{\mathcal{T}} \mathrm{d} \tau C_{\text {as }}(\tau, s)=\bar{C}(s)$

where

$$
C_{\text {as }}(\tau, s)=\lim _{K \rightarrow \infty} \frac{1}{K} \sum_{k=0}^{K} C(\tau+k \mathcal{T}, s) .
$$

Equations (25), (27)-(29) represent the generalized Green-Kubo relation for the diffusion coefficient of such periodically driven processes $x(t)$; notably, these per se constitute far from equilibrium processes.

\section{References}

[1] Hänggi P and Bartussek R 1996 Lect. Notes Phys. 476294

Jülicher F, Ajdari A and Prost J 1997 Rev. Mod. Phys. 691269

Porto M, Urbakh M and Klafter J 2001 J. Lumin. 94137

Astumian R D and Hänggi P 2002 Phys. Today 55 (11) 33

Reimann P and Hänggi P 2002 Appl. Phys. A 75169

Reimann P 2002 Phys. Rep. 36157

Linke H 2002 Appl. Phys. A 75167

Hänggi P, Marchesoni F and Nori F 2005 Ann. Phys., Lpz. 1451

[2] Jones P H, Goonasekera M and Renzoni F 2004 Phys. Rev. Lett. 93073904

[3] Lee S-H, Ladavac K, Polin M and Grier D G 2005 Phys. Rev. Lett. 94110601

[4] Babič D and Bechinger C 2005 Phys. Rev. Lett. 94148303

[5] Van de Vondel J, de Souza Silva C C, Zhu B Y, Morelle M and Moshchalkov V V 2005 Phys. Rev. Lett. 94 057003 
[6] Villegas J E, Gonzalez E M, Gonzalez M P, Anguita J V and Vincent J L 2005 Phys. Rev. B 71024519

[7] Péclet E 1841 Ann. Chim. Phys. 3107

Péclet E 1843 Traité de la Chaleur Considerée dans ses Applications 3 vols (Paris: Hachette)

[8] Jung P, Kissner J G and Hänggi P 1996 Phys. Rev. Lett. 763436

[9] Lindner B, Schimansky-Geier L, Reimann P, Hänggi P and Nagaoka M 1999 Phys. Rev. E 591417

[10] Hänggi P and Thomas H 1982 Phys. Rep. 88207

[11] Machura L, Kostur M, Talkner P, Łuczka J, Marchesoni F and Hänggi P 2004 Phys. Rev. E 70061105

[12] Landau L D and Lifshitz E M 1959 Fluid Dynamics (Oxford: Pergamon) p 203

[13] Suzuki D and Munakata T 2003 Phys. Rev. E 68021906

Wang H and Oster G 2002 Europhys. Lett. 57134

[14] Talkner P 2003 Physica A 325124

Talkner P, Machura L, Schindler M, Hänggi P and Łuczka J 2005 New J. Phys. 714

Casado-Pascual J, Gomez-Ordonez J, Morillo M, Lehmann J, Goychuk I and Hänggi P 2005 Phys. Rev. E 71 011101

[15] Fano U 1947 Phys. Rev. 7226

[16] Bartussek R, Hänggi P and Kissner J G 1994 Europhys. Lett. 28459

Savel'ev S, Marchesoni F, Hänggi P and Nori F 2004 Europhys. Lett. 67179

Savel'ev S, Marchesoni F, Hänggi P and Nori F 2004 Phys. Rev. E 70066109

Savel'ev S, Marchesoni F, Hänggi P and Nori F 2004 Eur. J. Phys. B 40403

[17] Mateos J L 2001 Acta Phys. Pol. B 32307

Mateos J L 2003 Physica A 32592

Arizmendi C M, Family F and Salas-Brito A L 2001 Phys. Rev. E 63061104

Larrondo H A, Family F and Arizmendi C M 2002 Physica A 30367

Larrondo H A, Arizmendi C M and Family F 2003 Physica A 320119

Son W-S, Kim I, Park Y-J and Kim C-M 2003 Phys. Rev. E 68067201

Barbi M and Salerno M 2000 Phys. Rev. E 621988

Borromeo M, Costantini G and Marchesoni F 2002 Phys. Rev. E 65041110

Sengupta S, Guantes R, Miret-Artes S and Hänggi P 2004 Physica A 338406

[18] Schreier M, Reimann P, Hänggi P and Pollak E 1998 Europhys. Lett. 44416

Costantini G and Marchesoni F 1999 Europhys. Lett. 48491

Reimann P, Van den Broeck C, Linke H, Hänggi P, Rubi J M and Perez-Madrid A 2001 Phys. Rev. Lett. 87 010602

Reimann P, Van den Broeck C, Linke H, Hänggi P, Rubi J M and Perez-Madrid A 2002 Phys. Rev. E 65031104

[19] Porto M, Urbakh M and Klafter J 2000 Phys. Rev. Lett. 85491 